\title{
Can Uncontrolled Blood Sugar Trigger Early Presentation of a Long Term Diabetic Complication? A Case Report of Lymphocytic Mastitis in a Recently Diagnosed Diabetic Patient
}

\section{Zainab Aldawood* and Jamila Alazhri}

Breast and Endocrine Section, Department of surgery, King Fahd Specialist Hospital,

Dammam, Saudi Arabia

*Corresponding Author: Zainab Aldawood, Breast and Endocrine Section, Department of surgery, King Fahd Specialist Hospital, Dammam, Saudi Arabia.
Received: June 18, 2021

Published: July 13, 2021

(C) All rights are reserved by Zainab

Aldawood* and Jamila Alazhri.

\begin{abstract}
Introduction: Lymphocytic mastitis is a condition associated with long term history of insulin dependent diabetes mellitus. It commonly presents as a painless breast mass that can imitate breast cancer both clinically and radiologically. The early recognition of this disease is crucial for appropriate management, especially in occasions where the presentation is unusual and least expected.

Presentation of Case: We report a case of a 32 years old female with a short-standing uncontrolled non- insulin dependent diabetes mellitus and multiple endocrine neoplasm syndrome type I [MEN1], who presented with Lymphocytic mastitis suspicious for breast cancer.

Discussion: The patient had a short history (5 years) of DM compared to the duration described in the literature, and unlike the previously reported cases, her DM was noninsulin dependent and she had MEN 1 syndrome. To our knowledge, no prior reports of lymphocytic mastitis associated with MEN syndrome has been published. We hypothesize that the long term histopathological effect of glycosylation in DM can be accelerated by persistently elevated blood sugar (uncontrolled DM) over a shorter period of time.

Conclusion: Lymphocytic mastitis was believed to be associated with long term history of IDDM. We recommend that LM be considered as a differential diagnosis of a breast mass in patients with IDDM as well as non-IDDM. The history of uncontrolled DM, even for short term, should trigger early suspicion of LM to avoid mismanaging this benign condition as breast cancer. Patients with DM, especially uncontrolled, should be encouraged to periodically examine their breasts for the signs of LM.
\end{abstract}

Keywords: Lymphocytic Mastitis; Fibrotic Mastopathy; Diabetic Mastopathy; Breast Cancer; Insulin Dependent Diabetes Mellitus

\section{Abbreviations}

LM: Lymphocytic Mastitis; MEN1: Neoplasm Syndrome Type I; IDDM: Insulin Dependent Diabetes Mellitus; DM: Diabetes Mellitus

\section{Introduction}

Lymphocytic mastitis (LM), also known as fibrotic mastopathy or diabetic mastopathy is a relatively rare condition [1]. Only case reports and small case series have been identified in the literature. LM usually presents in women with long term history of insulin dependent diabetes mellitus [2]. Nevertheless, it can be also present with other autoimmune conditions, for example, Rheumatoid arthritis, Hashimoto's thyroiditis and Sjögren's syndrome [3]. The etiology of LM is not well understood. One theory indicates that the use of exogenous insulin can induce inflammatory and immunological reactions [4]. However, another theory indicates that hyperglycemia may alter the extracellular matrix, by the process of glycosylation. This process stimulates the growth factors to induce the production of a collagen that is resistant to degradation [5]. The clinical and radiological presentation of LM can mimic breast 
cancer, and pathological confirmation with a biopsy is crucial to differentiate between the two entities and appropriately plan the management $[6,7]$.

\section{Case Presentation}

We present a case of a 32 years old female patient with a fiveyear history of uncontrolled non-insulin dependent diabetes mellitus and neuroendocrine pancreatic tumor, hyperprolactinoma and hyperparathyroidism [MEN I syndrome]. The patient was referred to our breast surgical oncology clinic with a painless right breast mass for 3-week duration. There was no history of skin changes or nipple discharge. She reported no history of oral contraceptive use, and no personal or family history of breast cancer. She is a mother of 1 child with a history of two miscarriages and no lactation history. On examination, there was a right breast firm mass, at the retroareolar area, measuring $2 \times 2$ centimeters $(\mathrm{cm})$ in size with no skin or nipple abnormality. Left breast and bilateral axillary examination were unremarkable. Ultrasound scan of the breast was performed and showed a round hypoechoic lesion with posterior acoustic shadow at the right 12 o'clock retroareolar region, it measured $1.4 \mathrm{~cm} \times 0.7 \mathrm{~cm}$ in size. The possibility of a complex hematoma, or a solid lesion was considered, BIRAD 4a, low level of suspicion for malignancy, and biopsy was recommended (Figure 1). A tru-cut biopsy of the suspicious lesion showed parenchymal fibrosis with dense lymphocytic infiltration, but no evidence of malignant cells. These findings were compatible with lymphocytic mastitis. The patient was reassured and managed conservatively with regular follow up in breast clinic.

Two weeks later, she presented to the emergency room with painful right breast lump. The lump was progressively increasing in size and the pain was not relieved by simple analgesia. There was no history of trauma or fever. Clinically, she looked well with normal vital signs and a body temperature of $37.4^{\circ} \mathrm{C}$. Breast examination, revealed firm right breast retro-areolar mass measuring 6 $\mathrm{x} 5 \mathrm{~cm}$ in diameter with erythema of the overlying skin (Figure 2). Blood work up showed WBC count of $12.8 \mathrm{mcl}$ with $79.3 \%$ neutrophils, she had elevated blood glucose level of $16 \mathrm{mmol} / \mathrm{L}$ and elevated HgbA1c level of $13.3 \mathrm{mmol} / \mathrm{L}$. Breast ultrasound showed heterogeneous complex mass, cystic in nature with echogenic component measuring $8.3 \times 5.9 \mathrm{~cm}$ at right breast retro-areolar region with parenchymal edema and thickening of the overlying skin (Figure 3). The ipsilateral axilla revealed multiple enlarged lymph nodes with preserved fatty hilum. The clinical and radiological impression was right breast abscess. The patient was admitted to the hospital for incision and drainage of the right breast abscess. Approximately $30 \mathrm{cc}$ of pus was drained and sent for culture. Tissue from the abscess cavity was excised and sent for histopathology examination.

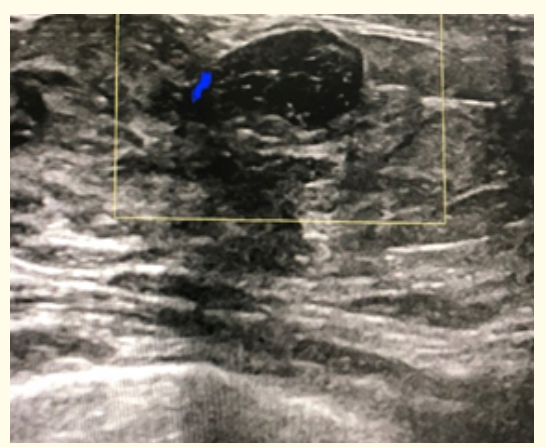

Figure 1: Ultrasound image of the right breast 12:00 retro-areolar region, showing a birads 4 a lesion.

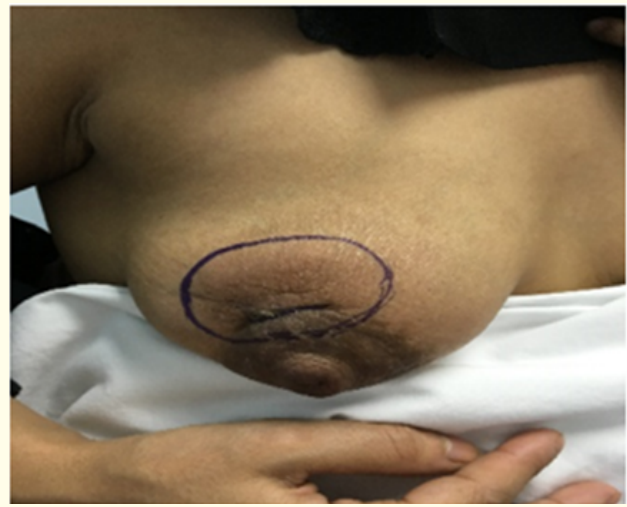

Figure 2: Right breast showing a 6x5 cm periareolar mass with overlying skin erythema.

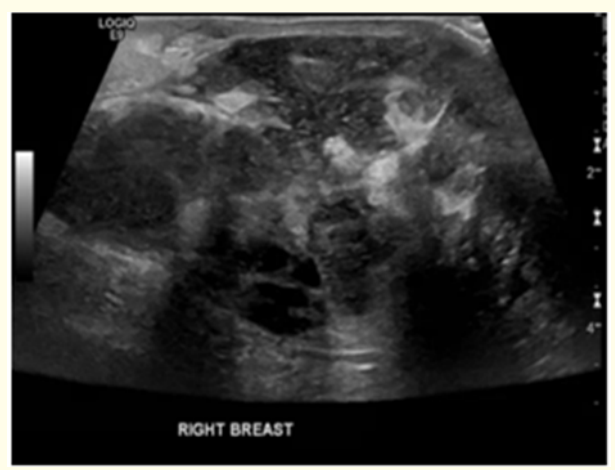

Figure 3: Ultrasound image of the right breast retroareolar area showing a heterogeneous complex predominantly cystic mass consistent with an abscess. 
Postoperatively, the patient was started on Cloxacillin $500 \mathrm{mg}$ I.V Q8h, and analgesics. Daily dressing of the breast wound was performed. Endocrine consultation was obtained and her blood sugar was controlled with Insulin Lantus 14 IU SQ daily and Insulin Regular 25 IU SQ TID. The patient had a smooth postoperative period. All local inflammatory signs resolved (Figure 4). Her WBC count dropped to $6.4 \mathrm{mcl}$. The final histopathology report revealed: lymphocytic mastitis with secondary acute mastitis/abscess, negative for malignancy. Result of the culture showed Staphylococcus epidermidis, sensitive to Cloxacillin. The patient was discharged home with oral analgesics and antibiotic. During the out-patient follow up, her wound healed completely within two weeks.

The patient was followed for 3 years period with no evidence of new breast lesions. In the interim, she underwent pancreatectomy, splenectomy, cholecystectomy with hepaticojejunostomy and gastrojejunostomy for the treatment of pancreatic neuroendocrine tumor. Her blood sugar level was controlled by Insulin Aspart 20 IU SQ TID and Insulin Detemir 10 IU SQ Daily, and her latest random blood sugar was $4 \mathrm{mmol} /$ Liter. The most recent follow up breast ultrasound showed a stable 1.5 centimeter, circumscribed mass at 12 o'clock in the right breast, with benign sonographic characteristics, BIRADS II (Figure 5).

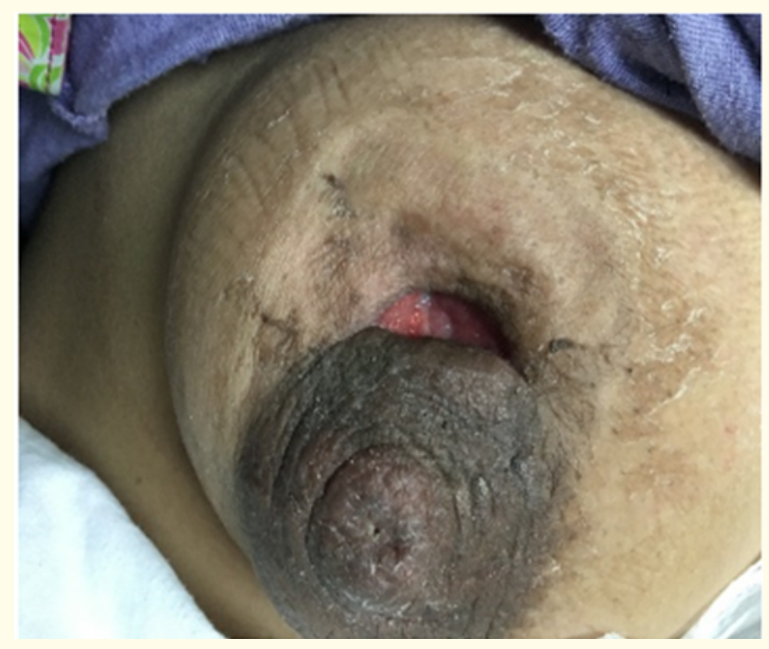

Figure 4: Right breast four days after incision and drainage of breast abscess, showing the resolution of inflammatory signs.

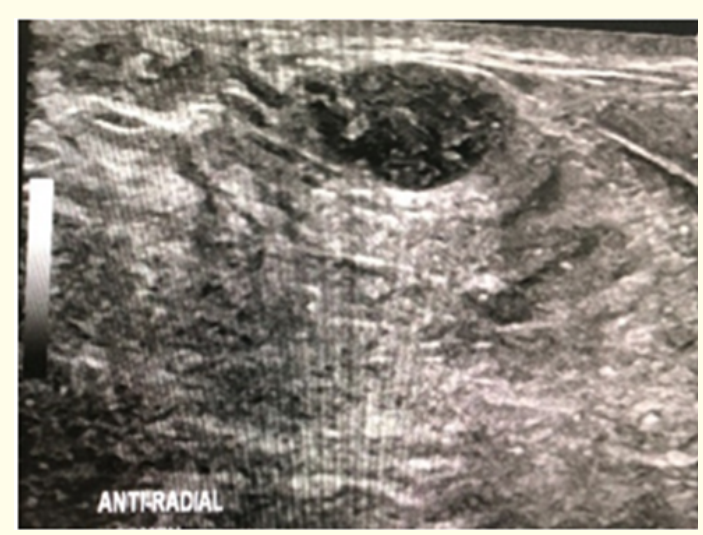

Figure 5: Most recent ultrasound image of the right breast showing a stable mass at 12 o'clock with benign characteristics, BIRADS II.

\section{Discussion}

Lymphocytic mastitis is a nonmalignant entity of the breast. However, its main differential diagnosis is breast cancer [3]. LM has been found in long standing noninsulin dependent diabetes mellitus, but less commonly than in insulin dependent diabetes mellitus [6]. In present of this strong relation between insulin dependent diabetes mellitus and lymphocytic mastitis, a hypothesis developed suggesting that LM can be an autoimmune disease as well [8]. LM has been also reported with other autoimmune diseases, such as Hashimoto's thyroiditis and systemic lupus erythematosus [6], and this is another relation suggesting an autoimmune cause However, LM can also present in patient without autoimmune diseases, neither diabetes mellitus [9].

In 1992, Tomaszewski., et al. reported a mean duration of more than 13 years from the diagnosis of diabetes mellitus for LM to develop. They also reported that the disease can be seen rarely in males and it is not limited to females [9]. In the present case, our patient had a short history (5 years) of DM compared to the duration described in the literature, and unlike the previously reported cases, her DM was noninsulin dependent and she had MEN 1 syndrome. To our knowledge, no prior reports of lymphocytic mastitis associated with MEN syndrome has been published.

The etiology of this disease is not clearly known. In 1985, Sternberg., et al. found that the glycosylation and increased intermo- 
lecular cross linkages in diabetics cause collagen to be resistant to degradation. This leads to the accumulation of connective tissue characteristic of some connective tissue disorders in diabetics, including mastopathy [10]. According to this data, we hypothesize that the long term histopathological effect of glycosylation in DM can be accelerated by persistently elevated blood sugar (uncontrolled DM) over a shorter period of time. This might explain the development of LM so early in our patient.

Clinically, most patients present with firm painless breast masses that are of variable sizes $[10,11]$. These masses can be located anywhere in the breast, but there is predominance in the subareolar region $[4,12,13]$, similar to our case presentation. With the disease progression it can also present with bilateral or multicentric involvement as reported in the literature [14].

In 1989, Logan., et al. established specific criteria for the diagnosis of LM, i.e. a long-term history of IDDM, firm palpable masses, mammographic dense glandular tissue and strong ultrasound acoustic shadowing [7]. However, these criteria still cannot distinguish LM from breast cancer since a firm mass with a posterior acoustic shadow on ultrasound is also suspicious for malignancy, as was labeled in our patient. Mammographic dense glandular tissue is actually a risk factor for breast cancer [15], and hence, cannot be used as a diagnostic criteria for LM. Our patient was 32 years old, and therefore, mammogram was not part of her initial workup. Although the long term insulin dependent diabetes mellitus is an important history to lower the threshold for the diagnosis of LM, it does not seem to be a constant factor. The patient we presented had a short term DM, and it was not insulin-dependent. Insulin levels and insulin resistance correlate with breast fat tissue heterogeneity, but their role in breast pathology remains unclear [16].

Histologically, lymphocytic infiltration of the breast tissue has been found predominantly to be of B lymphocytes in diabetic patient similar to other autoimmune diseases, while mainly of T lymphocytes in non-diabetic patients [3].

Shaffrey., et al. [17] compared breast biopsy results in patient with long term insulin dependent diabetes mellitus and those who have no diabetes mellitus history, same pathological findings were present in both including: lymphocytic lobulitis and ductitis, lymphocytic vasculitis, dense keloid-like fibrosis. However, epithelioid fibroblast was noticed only in the biopsy of insulin dependent diabetes mellitus patient which is the key distinguishing factor $[18,19]$.

The diagnosis of LM should be based on the combination of various information, including history, clinical examination and radiological findings. However, the diagnosis can only be confirmed in most cases by a tissue biopsy. This is particularly important to differentiate LM from breast cancer when the breast lesion is suspicious on radiological exams. The absence of long term history of insulin dependent diabetes mellitus, or the presence of non-insulin dependent diabetes mellitus should not exclude LM from the differential diagnosis. This may result in over investigating the patient and subjecting her to unnecessary surgical procedures like lumpectomy or mastectomy due to the fear of missing a breast cancer.

The favorable treatment of LM is conservative. Reassurance is very important to relieve the patient's anxiety and fear of breast cancer. Unnecessary surgical interventions in those patients has been reported to result in complications such as delay wound healing, wound infection, exacerbation the condition, and poor cosmetic outcome $[5,20,21]$. The treatment of LM should be focused on conserving the breast, controlling the serum blood sugar, and limiting surgical intervention to drainage of large breast abscesses that cannot be treated using needle aspiration and antibiotics. We believe that the tight control of serum blood sugar in our patient was the key behind her cure.

\section{Conclusion}

In conclusion, LM was believed to be associated with long term history of IDDM. We recommend that LM be considered as a differential diagnosis of a breast mass in patients with IDDM as well as non-IDDM. The history of uncontrolled DM, even for short term, should trigger early suspicion of LM to avoid mismanaging this benign condition as breast cancer. Patients with DM, especially uncontrolled, should be encouraged to periodically examine their breasts for the signs of LM.

\section{Conflict of Interest}

No conflict of interest.

\section{Bibliography}

1. Soler N and Khardori R. "Fibrous disease of the breast, thyroiditis, and cheiroarthropathy in type I diabetes mellitus". The Lancet 323.8370 (1984): 193-195. 
2. Campos G C P., et al. "Lymphocytic mastopathy mimicking breast malignancy: a case report". Radiologia Brasileira 47.4 (2014): 256.

3. Bilir B E., et al. "A metabolic syndrome case presenting with lymphocytic mastitis". Breast Care 7.6 (2012): 493-495.

4. Selma RV., et al. "Mastopatía diabética: clínica, hallazgos radiológicos y anatomopatológicos y tratamiento". Radiología 53.4 (2011): 349-354.

5. Camuto PM., et al. "Diabetic masthopaty. A report of 5 cases and a review of the literature". Archives of Surgery 135 (2000): 1190-1193.

6. Neetu G., et al. "Diabetic mastopathy: a case report and literature review". Case Reports in Oncology 3.2 (2010): 245-251.

7. Logan W W and Hoffman N Y. "Diabetic fibrous breast disease". Radiology 172.3 (1989): 667-670.

8. Chan H H L., et al. "Lymphocytic mastitis and rheumatoid arthritis". Journal Hong Kong College of Radiologists 5 (2002): 233-234.

9. Tomaszewski JE., et al. "Diabetic mastopathy: a distinctive clinicopathologic entity”. Human Pathology 23.7 (1992): 780-786.

10. Sternberg M., et al. "Connective tissue in diabetes mellitus: biochemical alterations of the intercellular matrix with special reference to proteoglycans, collagens and basement membranes". Diabete and Metabolisme 11.1 (1985): 27-50.

11. Pereira M A D Q F., et al. "Fibrous mastopathy: clinical, imaging, and histopathologic findings of 31 cases". Journal of Obstetrics and Gynaecology Research 36.2 (2010): 326-335.

12. Alhabshi S M I., et al. "Lymphocytic mastitis mimicking breast carcinoma, radiology and pathology correlation: review of two cases". The Malaysian Journal of Medical Sciences: MJMS 20.3 (2013): 83.

13. Williams P H., et al. "Sclerosing lymphocytic lobulitis of the breast”. Clinical Radiology 50.3 (1995): 165-167.

14. Bayer U., et al. "Bilateral, tumorlike diabetic mastopathy-progression and regression of the disease during 5-year follow up". European Journal of Radiology 26.3 (1998): 248-253.
16. Rzymski P., et al. "Correlation between insulin resistance and breast elasticity heterogeneity measured by shear wave elastography in premenopausal women-a pilot study". Archives of Medical Science: AMS 7.6 (2011): 1017.

17. Shaffrey JK., et al. "Diabetic fibrous mastopathy: case reports and radiologic-pathologic correlation". Breast Journal 6.6 (2000): 414-417.

18. Sabate JM., et al. "Radiologic evaluation of uncommon inflammatory and reactive breast disorders". Radiographics 25.2 (2005): 411-424.

19. Andrews-Tang DA., et al. "Diabetic mastopathy: adjunctive use of ultrasound and utility of core biopsy in diagnosis". Breast Journal 6.3 (2000): 183-188.

20. Goulabchand R., et al. "Mastitis in Autoimmune Diseases: Review of the Literature, Diagnostic Pathway, and Pathophysiological Key Players". Journal of Clinical Medicine 9.4 (2020): 958.

21. Sankaye S and Kachewar S. "Diabetic mastopathy". The Australasian Medical Journal 5.6 (2012): 296.

\section{Volume 5 Issue 8 August 2021 \\ (C) All rights are reserved by Zainab Aldawood and Jamila Alazhri.}

15. "What Does It Mean to Have Dense Breasts?" (2020). 